\title{
Tonic immobility in chicks during presentations and withdrawals of an imprinting stimulus
}

\author{
PETER V. BERNS and LAURA M. BELL \\ University of Pennsylvania, Philadelphia, Pennsylvania 19104
}

\begin{abstract}
We investigated the effects of presentation and withdrawal of an imprinting stimulus on durations of the tonic immobility reaction in domestic fowl. In Experiment 1, presentation and withdrawal of the imprinting stimulus was compared to similar manipulations with a novel stimulus. Differences in the immobility reaction were due to specific effects of the imprinting stimulus. In Experiment 2, we demonstrated the effect of the presence or withdrawal of only the imprinting stimulus on the duration of the immobility reaction. The presence of the imprinting stimulus shortened the duration of the immobility reaction, while the withdrawal of the same stimulus lengthened the duration of the reaction, relative to a baseline condition. The results are interpreted in terms of central affective states which interact in simple ways.
\end{abstract}

Central affective states are thought to play an important role in the control of behavior; yet, the existence of such states is purely inferential, a product of the psychologist's analysis of the sudden changes occurring in ongoing behavior when pleasant and unpleasant stimuli are introduced and then terminated. One can begin to give empirical status to such inferred affective states by studying the interactions caused when two such states are elicited simultaneously. Thus, an affective state becomes that state aroused by stimulus $X$ which, when combined with the state aroused by stimulus $Y$, yields a specific behavioral phenomenon, $Z$. This is the strategy represented by our experiments. We arouse in chicks either the affective state controlled by the presence of an imprinting object (pleasant) or that controlled by the removal of an imprinting object (aversive) and ask what happens to the tonic immobility reaction induced by forced restraint (aversive) as a consequence of the interactions of the affective states controlled by the three stimulus complexes.

Chicks and ducklings manifest a characteristic pattern of response to presentations and withdrawals of an imprinting stimulus. In the presence of the imprinting object, the animal seems content and will follow the stimulus avidly, emitting few distress vocalizations (Hoffman \& Solomon, 1974). Withdrawal of that same stimulus dramatically changes the animal's behavior. The hatchling will actively search for the stimulus, while emitting high-intensity distress

This research was supported by Grant MH-29187 to Richard L. Solomon. The authors would like to thank Richard L. Solomon, Mark D. Starr, David R. Burdette, and David Hildebrand for their helpful comments on this paper. Requests for reprints should be sent to Richard L. Solomon, Department of Psychology, University of Pennsylvania, Philadelphia, Pennsylvania 19104. calls. Either the passage of time or reintroduction of the imprinting stimulus will decrease the distress reaction.

There is evidence that the presence of an imprinting object is positively reinforcing or pleasurable. Hoffman and Ratner (1973), using distress-calling as an index of the duckling's affective state, demonstrated that mere presentation of the imprinting stimulus suppressed distress-calling. Further evidence in support of this finding comes from experiments showing that presentation of an imprinting stimulus can strengthen an operant response (Eiserer \& Hoffman, 1973; Hoffman, Searle, Toffee, \& Kozma, 1966). In addition, there are aversive aftereffects when exposure to an imprinting stimulus is terminated. Hoffman and Ratner (1973) demonstrated that withdrawal of an imprinting stimulus elicited a significant amount of distress-calling in ducklings which emitted no distress vocalizations while in the presence of the same stimulus. If withdrawal of the imprinting stimulus is negatively reinforcing, it should weaken the probability of a contingent response. Hoffman, Stratton, and Newby (1969) found that ducklings would refrain from following the imprinting stimulus when brief stimulus termination was a consequence of following. Thus, it seems reasonable to conclude that the presence of the imprinting stimulus is positively reinforcing, and probably pleasurable, while its absence is negatively reinforcing, and probably aversive.

Chicks manifest a distinct reaction to firm, physical restraint. Initially, they will struggle vigorously while being restrained, but if restraint is continued, a stereotypic response occurs, "tonic immobility," characterized by a rigid posture in which the bird's head is directed downward and its legs either extended or held next to the body (Ratner, 1967). The muscula- 
ture is hypertonic. Both catatonic-like waxy flexibility and Parkinsonian-like tremors may be observed in the extremities. Heart rate increases, while respiration becomes faster. If the immobilized animal is undisturbed, the immobility reaction dissipates and the animal rights itself. The reaction may be disrupted by the abrupt onset of an intense stimulus. Although there does not seem to be a specific behavioral predictor of immobility termination, the occurrence of vocalizations and the return of heart rate to baseline levels often precede the right response.

Tonic immobility is probably mediated by a central affective state of an aversive sort, a consequence of the prey-predator system. If so, manipulations of the chick's affective state by reinforcing stimulation might affect the immobility reaction. Gallup and others have extensively investigated this possibility in recent years. They have found that the manipulations assumed to increase fear result in increased susceptibility to, and durations of, tonic immobility. The opposite result has been obtained for manipulations assumed to decrease fear. Those procedures which increase the novelty of a testing situation (by introducing a novel experimenter, novel testing procedure, or novel stimuli) enhance the duration of the immobility response (Gilman, Marcuse, \& Moore, 1950). Conversely, decreases in the novelty of the testing situation (by extensive handling of the animal or repeated elicitation of the immobility response) shorten the duration of the immobility reaction (Gilman, Marcuse, \& Moore, 1950; Nash \& Gallup, 1976; Ratner \& Thompson, 1960). Similarly, the preinduction administration of aversive auditory (Gallup, 1974) and tactile stimulation (Gallup, Creekmore, \& Hill, 1970; Gallup, Nash, Potter, \& Donnegan, 1970) result in increased durations of, and susceptibility to, the immobility reaction; this increase is proportional to the intensity of stimulation presented. Finally, chemical manipulations designed to alter the animal's responsivity to stimulation have an effect on immobility. Administration of Pacitran (Gallup, Nash, \& Brown, 1971), a tranquilizer known to reduce emotionality in domestic fowl, results in lower susceptibility to, and shorter durations of, tonic immobility. Administration of adrenalin (Braud \& Ginsburg, 1973) results in greater durations of immobility. Such findings are quite compatible with an interpretation of tonic immobility that stresses the role of central affective states in its control.

Our experiments demonstrate the role that central affective states play in the control of both tonic immobility and imprinting. If central affective states mediate immobility, and the presentation or withdrawal of an imprinting stimulus alters these states, the duration of the immobility reaction should be affected. In Experiment 1, with a within-subjects design, the presence and absence of a novel stimulus served to clarify whether differences in the manifes- tations of immobility are specific to the imprinting stimulus. In Experiment 2, the nature of these differences was studied with a between-subjects design.

\section{EXPERIMENT 1}

\section{Subjects \\ Method}

Nineteen White Leghorn domestic fowl (Gallus gallus) were used. Fertilized eggs were obtained from George Shaw Hatchery, Media, Pennsylvania, at 18 days. They were hatched communally in a darkened incubator. The hatchlings remained in the incubator until the first imprinting session, and then were housed for the remainder of the experiment in individual cages on a 12-h lightdark cycle, with ad-lib food and water.

\section{Apparatus}

The experimental chamber consisted of subject and stimulus compartments, separated by a fine mesh screen. The subject compartment was approximately $12 \times 30 \mathrm{in} .(30.5 \times 76.2 \mathrm{~cm})$ and it was lined with Sani-cell animal bedding. The imprinting stimulus was a taxidermically prepared adult female duck that moved in a semicircular path at a speed of approximately $30 \mathrm{~cm} / \mathrm{sec}$. Presentation of the imprinting stimulus involved illumination of the stimulus compartment, movement of the imprinting stimulus, and introduction of a low-intensity tone, interrupted three times a second.

Novel stimulus presentation involved illumination of a flashing 40-W bulb located in the corner of the subject compartment, and a low-intensity, pure tone emanating from a speaker mounted directly behind the bulb.

\section{Procedure}

Phase 1: Imprinting sessions. Approximately 12 to $16 \mathrm{~h}$ posthatch, each animal was placed individually in the subject compartment. After $40 \mathrm{sec}$ adaptation, imprinting began. Stimulus presentation was broken into two 5 -min periods, separated by a 2 -min interstimulus interval. Upon termination of the second stimulus presentation, the animal remained in the apparatus for an additional $2 \mathrm{~min}$. Chicks were exposed to the imprinting stimulus for $10 \mathrm{~min}$ on each of 7 consecutive days.

Phase 2: Test sessions. A standard procedure was followed for inducing immobility. The experimenter restrained the animal on its back, with its legs extended. Restraint was continued until the immobility reaction occurred. Then, as the animal ceased struggling, the experimenter would slowly withdraw his hand. The duration of the immobility was measured by stopwatch.

The test phase included five stimulus conditions. (1) Baseline (BASE)-Two minutes after placing the animal in the subject compartment, the experimenter induced immobility. Termination of the reaction was self-paced, and the animal was confined to the experimental chamber for $15 \mathrm{sec}$ following termination. (2) Imprinting stimulus present (IS-ON)-After the animal was placed in the subject compartment, stimulus presentation occurred as described in Phase 1. However, 2 min into the second stimulus exposure period, the immobility induction procedure was administered. The imprinting stimulus remained present throughout the duration of the immobility reaction and for an additional $15 \mathrm{sec}$ following termination. (3) Imprinting stimulus withdrawal (IS-OFF) -Once again, the standard procedure for stimulus presentation was initiated $30 \mathrm{sec}$ after the subject was placed in the experimental chamber. Immobility was induced $2 \mathrm{~min}$ after the termination of the second stimulus presentation. The animal remained in the apparatus for $15 \mathrm{sec}$ following termination of the reaction. (4) Novel stimulus present (NOV-ON) and (5) Novel stimulus withdrawal (NOV-OFF)-The procedures involved in these test conditions were identical to those described for the IS-ON and IS-OFF treatments, with one exception: the novel stimulus was employed in place of the imprinting stimulus. 


\section{Results}

The duration of restraint required to induce immobility did not vary as a function of the experimental treatment condition $(p>.10)$. An analysis of variance of duration of immobility indicated a significant effect of treatment $[F(1,136)=5.21, p<.01]$. Since there were no significant differences in duration of immobility across measures of each stimulus condition (Baseline ${ }_{1}$ vs. Baseline ${ }_{2}$, IS-ON ${ }_{1}$ vs. IS-ON $\mathrm{ON}_{2}$, IS$\mathrm{OFF}_{1}$ vs. IS-OFF 2 ), these trials were combined.

For all subjects, the duration of immobility during the presence of an imprinting stimulus was significantly less than that during the baseline condition $[\mathrm{t}(38)=1.72, \mathrm{p}<.05]$. The duration of immobility during withdrawal of the same stimulus was significantly greater than that during the baseline condition $[\mathrm{t}(38)=1.84, \mathrm{p}<.05]$. In addition, the duration of immobility induced in the presence of a novel stimulus was significantly greater than that during the baseline condition $[t(19)=2.05, p<.025]$. The duration of immobility induced by withdrawal of the novel stimulus was not statistically different from that induced during the baseline condition.

\section{Discussion}

There was a clear difference in the duration of immobility as a function of the presence or absence of an imprinting stimulus. Relative to the baseline condition, these effects were opposite in direction: the presence of the imprinting stimulus attenuated the immobility reaction, while the withdrawal of that same stimulus augmented it. The differences between the imprinting stimulus and novel stimulus condition were specific effects engendered by the presentation and withdrawal of an imprinting stimulus.

\section{EXPERIMENT 2}

Experiment 2 investigated the effects of the presence or absence of an imprinting stimulus on the duration of tonic immobility, using a between-groups design rather than the within-subject design of Experiment 1 .

\section{Subjects and Apparatus}

\section{Method}

Fifty White Leghorn domestic fowl (Gallus gallus) were used as subjects. They were obtained and cared for as described in Experiment 1.

The apparatus was identical to that described in Experiment 1.

\section{Procedure}

Phase 1: Imprinting sessions. The procedure was identical to that described in Experiment 1. Two groups of animals participated in this experiment. Replication A included 20 subjects, while Replication B included 30 subjects.

Phase 2: Test sessions. Upon conclusion of Phase 1, all animals were arbitrarily separated among three experimental conditions. The experimental manipulations were the baseline (B) or contròl, imprinting stimulus presence (IS-ON), and imprinting stimulus withdrawal (IS-OFF) conditions, just as described in Experiment 1.
The between-group design of Experiment 2 controlled for any order effects that could hinder our interpretation of the data of Experiment 1. In addition, it controlled for developmental changes in the immobility reaction. Subjects in each treatment group received one trial daily on each of the 5 test days. The intertrial interval was approximately $24 \mathrm{~h}$.

The immobility-induction procedure in Experiment 2 was identical to that of Experiment 1.

\section{Results}

A four-way analysis of variance for duration of immobility indicated main effects for both experimenter $[F(1,192)=34.14, \mathrm{p}<.001]$ and treatment manipulation $[\mathrm{F}(1,192)=48.12, \mathrm{p}<.001]$. The existence of any other interaction or main effects was not confirmed. Specifically, the two groups studied in Replications A and B did not vary in immobility duration $[F(1,192)=.07]$, and the response did not vary as a function of test session $[\mathrm{F}(4,192)=1.16]$. Note that the Treatment-Experimenter interaction effects were not significant $[F(2,192)=.66]$. The differences in duration of immobility as a function of experimenter may have been due to subtle variations in the technique of induction, such as latency to restrain the animal, abruptness of inversion, and pressure applied during restraint. The treatment effects take the other sources of variance into account.

The duration of immobility following withdrawal of an imprinted stimulus was significantly greater than that during the control condition; IS-OFF mean $=55.7 \mathrm{sec}$, CONTROL mean $=41.9 \mathrm{sec}[\mathrm{t}(85)=$ $7.16, \mathrm{p}<.001]$. The duration of immobility during the presence of an imprinting stimulus was significantly suppressed; IS-ON mean $=15.6 \mathrm{sec}, \mathrm{CON}$ TROL mean $=41.9 \mathrm{sec}[\mathrm{t}(85)=2.06, \mathrm{p}<.025]$. Finally, a regression analysis suggested a significant degree of association between distress vocalizations in the last $60 \mathrm{sec}$ preceding immobility and the subsequent duration $[\mathrm{y}=1.8+.36 \mathrm{X} ; \mathrm{r}=.232 ; \mathrm{t}(166)$ $=3.07, \mathrm{p}<.01]$.

\section{DISCUSSION}

The presence or absence of an imprinting stimulus has a specific effect on the duration of tonic immobility in chicks. ${ }^{1}$ The effect can be explained by central affective states in control of the two behaviors. We can assume that the presence of an imprinting stimulus elicits an affective state which attenuates the state that controls the immobility reaction. Withdrawal of an imprinting stimulus engenders an affective state that is qualitatively different, hedonically opposite to the state elicited by the presence of the stimulus, resulting in enhanced durations of immobility.

Assume that tonic immobility is under the control of an aversive state. The withdrawal of an imprinting stimulus also engenders a hedonically aversive state. Enhancement of the immobility response following 
withdrawal of the imprinting stimulus then can be explained by adding together two aversive state intensities. On the other hand, the presence of an imprinting stimulus engenders a state that is hedonically pleasurable. Attenuation of the immobility duration is the result of subtraction of the pleasurable state intensity from the aversive state intensity. In commonsense terms, the hatchling responds to the experimenter as it would to other predators and is frightened when seized and immobilized. Normally, in the presence of the mother surrogate the hatchling is happy. When the chick is seized, presence of the mother surrogate effectively reduces the total aversiveness of affect, thus leading to relatively short durations of tonic immobility. However, when the mother surrogate is removed, the chick is normally lonely and distressed, and this aversive state combines with the fright accompanying immobilization to yield an augmented aversive affect. The increased duration of tonic immobility reflects this summation.

\section{REFERENCES}

Braud, W. G., \& Ginsburg, H. J. Effect of administration of adrenalin on the immobility reaction in domestic fowl. Journal of Comparative and Physiological Psychology, 1973, 83, 124-127.

Eise rer, L. A., \& Hoffman, H. S. Priming of duckling responses by presenting an imprinting stimulus. Journal of Comparative and Physiological Psychology, 1973, 82, 345-359.

Gallup, G. G. Animal hypnosis: Factual status of a fictional concept. Psychological Bulletin, 1974, 81, 836-853.

Gallup, G. G., Creekmore, H. S., \& Hill, W. E. Shockenhanced immobility reactions in chickens: Support for the fear hypothesis. Psychological Record, 1970, 20, 243-245.

Gallup, G. G., Nash, R. F., \& Brown, C. W. The effects of a tranquilizer on the immobility reaction in chickens: Additional support for the fear hypothesis. Psychonomic Science, $1971,23,127-128$.
Gallup, G. G., Nash, R. F., Potter, R. J., \& Donnegan, N. H. Effects of varying conditions of fear on immobility reactions in domestic chickens. Journal of Comparative and Physiological Psychology, 1970, 73, 442-445.

Gilman, T. J., Marcuse, F. L., \& Moore, A. U. Animal hypnosis: A study of the induction of tonic immobility in chickens. Journal of Comparative and Physiological Psychology, 1950, 43, 99-111.

Hoffman, H. S., \& RAtneR, A. M. A reinforcement model of imprinting: Implications for socialization in monkeys and men. Psychological Review, 1973, 80, 527-544.

Hoffman, H. S., Searle, J. L., Toffee, S., \& Kozma, F. Behavioral control by an imprinting stimulus. Journal of the Experimental Analysis of Behavior, 1966, 9, 177-189.

Hoffman, H. S., \& Solomon, R. L. An opponent-process theory of motivation: III. Some affective dynamics of imprinting. Learning and Motivation, 1974, 5, 149-164.

Hoffman, H. S., Stratton, J. W., \& Newby, V. Punishment by response-contingent withdrawal of an imprinted stimulus. Science, 1969, 163, 702-704.

Nash, R. F., \& Gallup, G. G. Habituation and tonic immobility in domestic chickens. Journal of Comparative and Physiological Psychology, 1976, 90, 870-877.

Ratner, S. C. Comparative aspects of hypnosis. In J. E. Gordon (Ed.), Handbook of clinical and experimental hypnosis. New York: Macmillan, 1967.

Ratner, S. C., \& Thompson, R. W. Immobility reactions of domestic fowl as a function of age and prior experience. Animal Behaviour, 1960, 8, 186-191.

\section{NOTE}

1. After completion of this experiment and submission of this report, the editors called our attention to a paper reporting that the presence of an imprinting stimulus could attenuate tonic immobility in chicks. See Hodges, S., \& Prestrude, A. M. Effects of an imprinting stimulus on tonic immobility in young domestic chicks. The Psychological Record, 1978, 28, 85-94.

(Received for publication April 21, 1978; revision accepted December 4,1978 .) 